\title{
Revisiting a model to predict pure triglyceride thermodynamic properties: parameter optimization and performance
}

\author{
Julia Seilert ${ }^{1}$, Arun Moorthy ${ }^{2}$, Anthony Kearsley ${ }^{3}$, and Eckhardt Flöter ${ }^{1}$ \\ ${ }^{1}$ Technical University of Berlin \\ ${ }^{2}$ National Institute of Standards and Technology Material Measurement Laboratory \\ ${ }^{3}$ National Institute of Standards and Technology Applied and Computational Mathematics \\ Division
}

May 4, 2021

\begin{abstract}
In 1990, a well-known model to predict pure component properties of triglycerides was presented by Wesdorp in "Liquid-multiple solid phase equilibria in fats: theory and experiments" and has been shown to perform well despite making thermodynamically inconsistent predictions for certain test cases. In this study, the underlying parameter set is improved to deliver more physically consistent predictions, i.e., increasing melting point and enthalpy of fusion with increasing stability of the polymorphs, without deterioration of the primary model quality to describe the available experimental data. Interestingly, when a curated dataset containing only thermodynamically consistent data is compared to a broader dataset, it appears that the model's efficacy is highly dependent on the quantity of data, specifically the number of unsaturated triglycerides data. Quality and thermodynamic consistency of model predictions and the condition of a reliable description of monoacid triglycerides as a subset is discussed, addressing a potential interdependence.
\end{abstract}

\section{Hosted file}

2604_Parameter_optimization_final_vs.pdf available at https://authorea.com/users/ 410557/articles/520725-revisiting-a-model-to-predict-pure-triglyceride-thermodynamicproperties-parameter-optimization-and-performance 


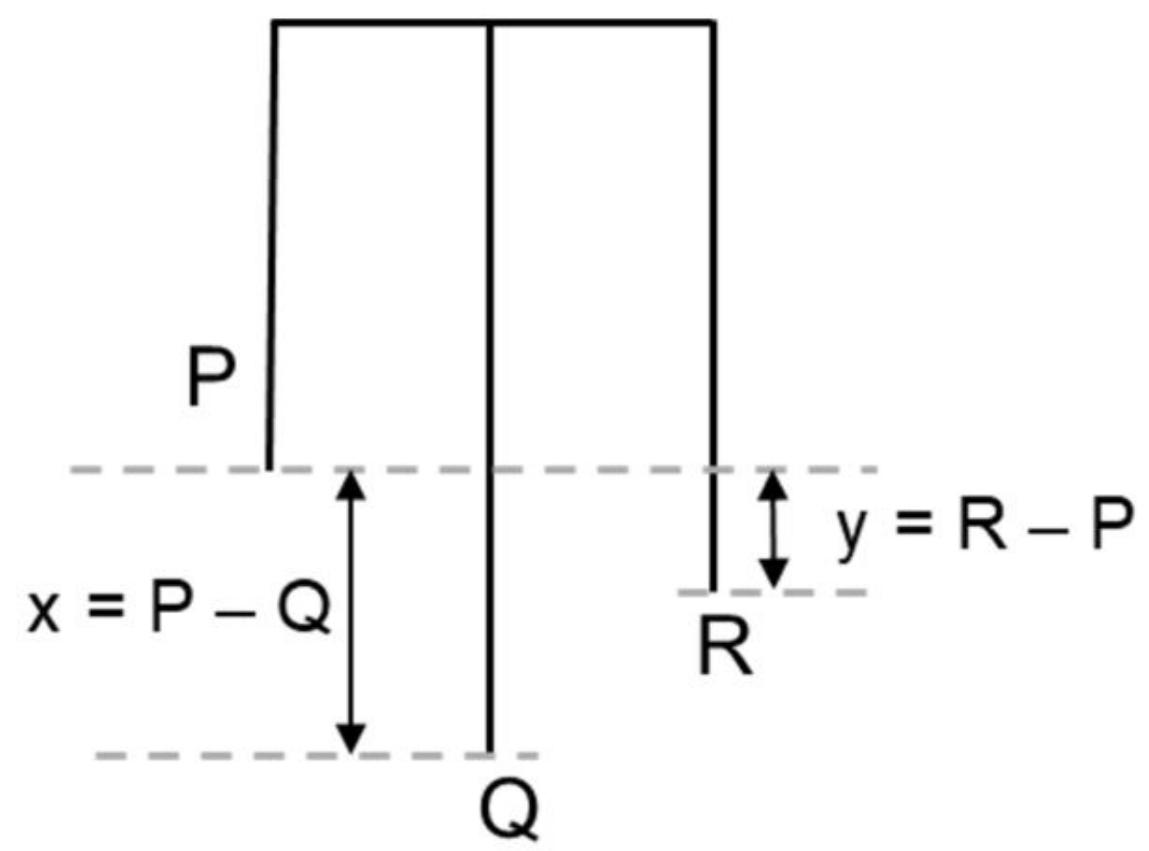




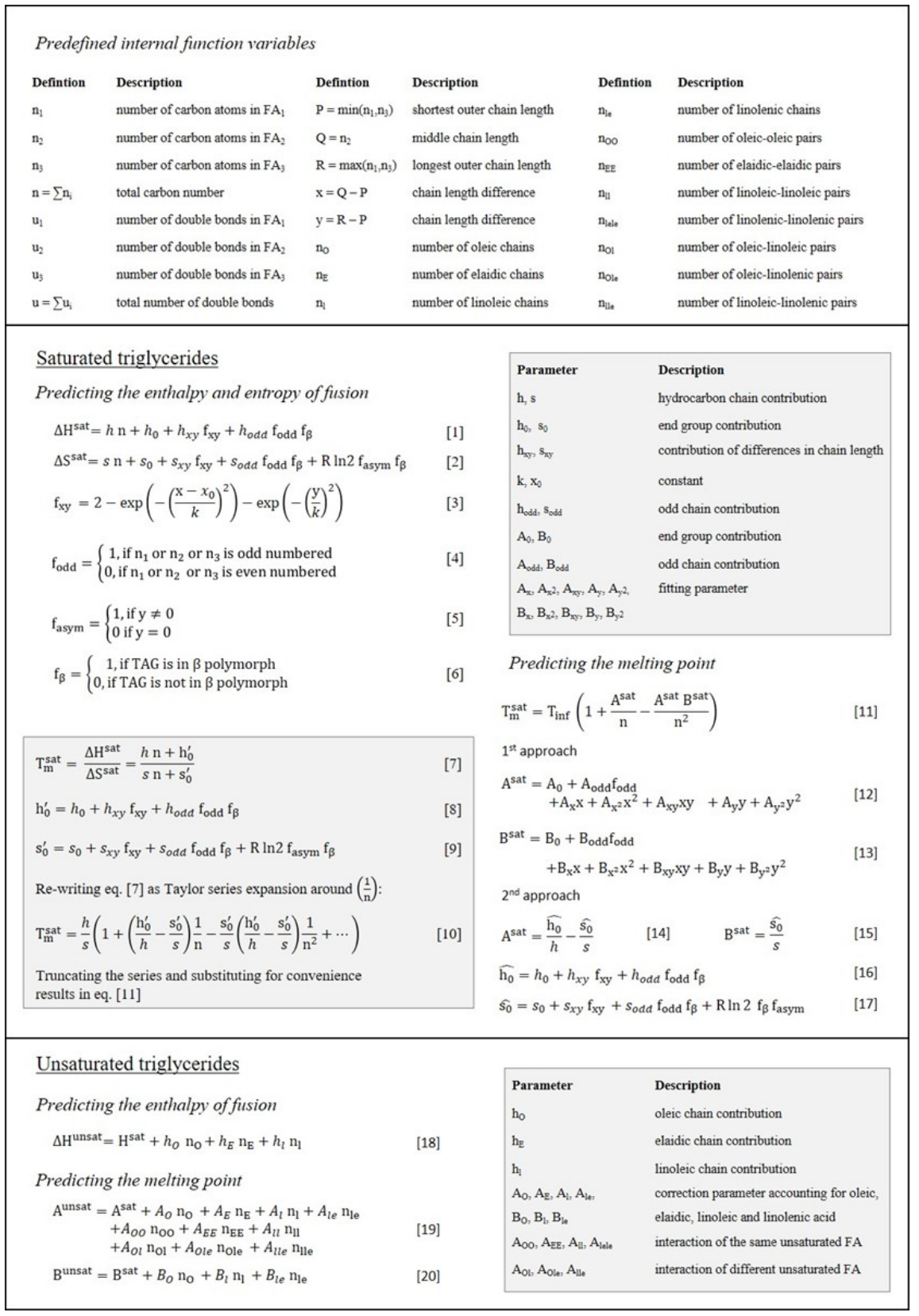



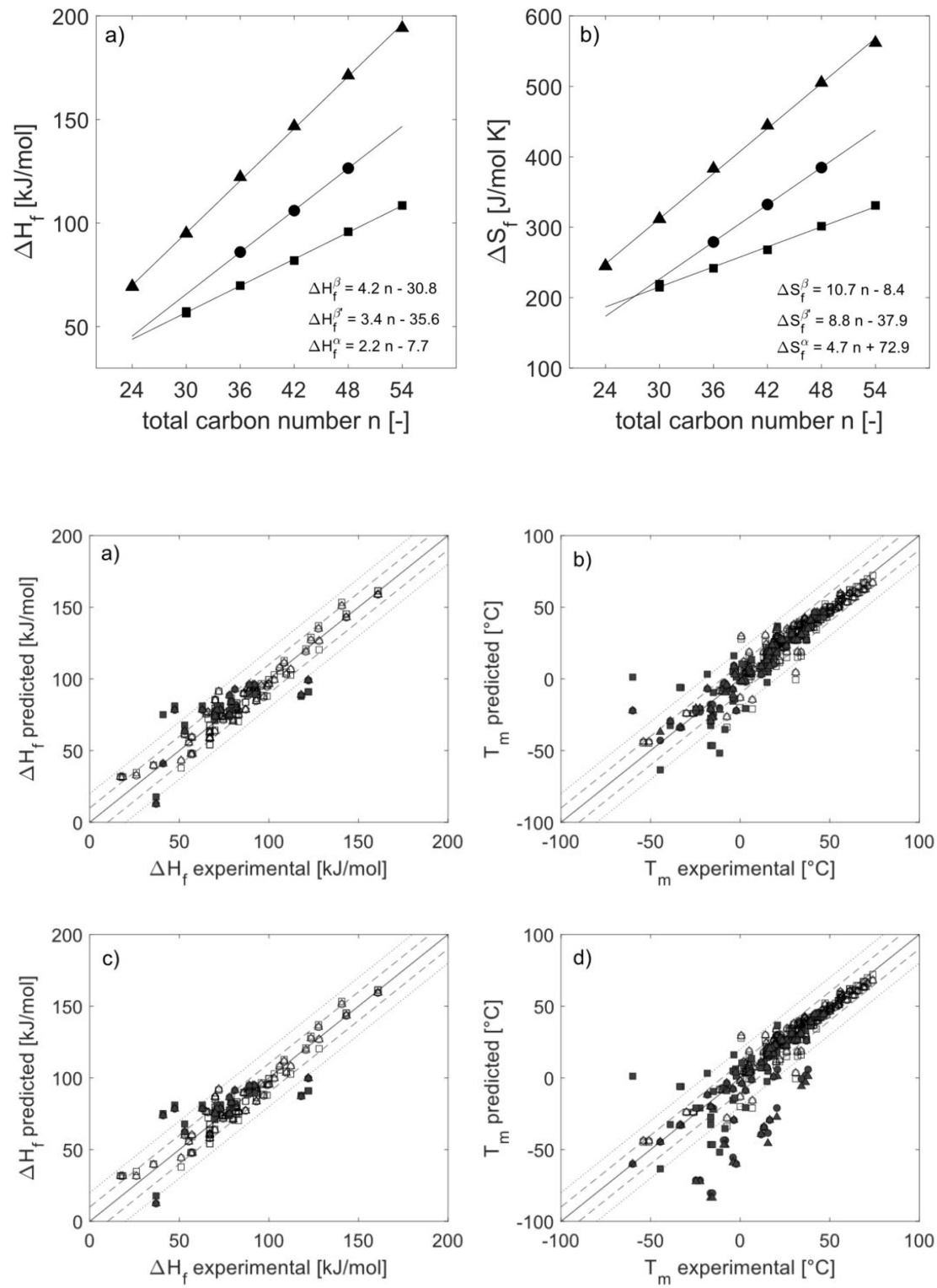